\title{
Caracterización de harinas y almidones de batatas (Ipomoea batatas Lam.) de la costa caribe colombiana
}

\section{Characterization of batatas flours and starches (Ipomoea batatas Lam.) from the colombian caribbean coast}

\author{
Karla Jiménez-Villalba ${ }^{1}$; Lina Arrieta-Banquet ${ }^{2}$; Jairo Salcedo-Mendoza ${ }^{3}$; Karen Contreras-Lozano ${ }^{4}$
}

1 Ing. Agroindustrial, Grupo de investigación en Procesos Agroindustriales y Desarrollo Sostenible (PADES), Facultad de Ingeniería. Universidad de Sucre, Sede Puerta Roja, Carrera 28 No. 5-267, Sincelejo, Sucre, Colombia, e-mail: jimenez.karla.20@gmail.com, (Dhttps://orcid.org/0000-0001-9630-2895

2 Ing. Agroindustrial, Grupo de investigación en Procesos Agroindustriales y Desarrollo Sostenible (PADES), Facultad de Ingeniería. Universidad de Sucre, Sede Puerta Roja, Carrera 28 No. 5-267, Sincelejo - Sucre, Colombia, e-mail: linaarrietabannquet@gmail.com, Dhttps://orcid.org/0000-0002-7154-1942

${ }^{3}$ Ing. Químico, Ph.D, Grupo de investigación en Procesos Agroindustriales y Desarrollo Sostenible (PADES), Facultad de Ingeniería. Universidad de Sucre, Sede Puerta Roja, Carrera 28 No. 5-267, Sincelejo, Sucre, Colombia, e-mail: jairo.salcedo@unisucre.edu.co, Dhttps://orcid.org/0000-0002-9901-9793

${ }^{4}$ Ing. Agroindustrial, Grupo de investigación en Procesos Agroindustriales y Desarrollo Sostenible (PADES), Facultad de Ingeniería. Universidad de Sucre, Sede Puerta Roja, Carrera 28 No. 5-267, Sincelejo, Sucre, Colombia, e-mail: kpcontrerasl@unal.edu.co, Dhttps://orcid.org/0000-0002-5237-1490

Cómo citar: Jiménez-Villalba, K.; Arrieta-Banquet, L.; Salcedo-Mendoza, J.; Contreras-Lozano, K. 2019. Caracterización de harinas y almidones de batatas (Ipomoea batatas Lam.) de la costa caribe colombiana. Rev. U.D.C.A Act \& Div. Cient. 22(1):e1185. https://doi. org/10.31910/rudca.v22.n1.2019.1185

Artículo de acceso abierto publicado por Revista U.D.C.A Actualidad \& Divulgación Científica bajo una licencia Creative Commons CC BY-NC 4.0

Recibido: Junio 14 de 2018

Aceptado: Febrero 24 de 2019

\section{RESUMEN}

El cultivo de batata en Colombia registra pocos excedentes para el mercado nacional, su rendimiento se encuentra en aproximadamente 5t/ha. Dicha producción, se centra en la región Caribe colombiana, en los departamentos de Córdoba y Sucre; sin embargo, el cultivo de la batata podría llegar a ocupar un lugar de importancia para la agroindustria, por los aportes nutricionales y por su alto contenido de almidón, considerado ideal para la obtención industrial de harinas y almidones. En esta investigación, se determinaron las propiedades estructurales y funcionales de harinas y almidones, obtenidos de cuatro variedades de batata, cultivadas en la región Caribe colombiana. Las propiedades funcionales analizadas fueron la capacidad de absorción de agua, amilosa, viscosidad máxima, temperatura de gelatinización, asentamiento, estabilidad y el comportamiento estructural, que fue estudiado mediante espectroscopia de infrarrojo FT-IR. Los resultados presentaron variaciones en los genotipos y las localidades evaluadas; los viscoamilograma en harina mostraron valores alentadores para la industria, tanto la viscosidad máxima $(300 \mathrm{cP})$ como el breakdown $(-15,64 \mathrm{cP})$ y setback $(186,65 \mathrm{cP})$, mientras los almidones presentaron valores relativamente altos, por lo cual, se recomienda realizar técnicas de modificación a la estructura nativa del almidón, modificaciones físicas, enzimáticas o químicas, para ampliar sus aplicaciones. El comportamiento estructural mostró señales que indican la presencia de grupos $\mathrm{OH}$, $\mathrm{CH}, \mathrm{CO}, \mathrm{H}_{2} \mathrm{O}$ en las harinas y almidones, de batata evaluados.

Palabras clave: genotipo; harina; almidón; viscoamilograma; espectroscopia.

\section{ABSTRACT}

The production of sweet potatoes in Colombia presents few surplus for the domestic market, its performance is approximately $5 \mathrm{t} / \mathrm{ha}$, with a sown area of barely 5 hectares; this production is obtained on few areas of the national agricultural region (Caribbean region, 
Jiménez-Villalba, K.; Arrieta-Banquet, L.; Salcedo-Mendoza, J.; Contreras-Lozano, K.: Harinas y almidones de batata

specifically in the departments of Córdoba and Sucre). However, the crop could reach an important place in the agro-industry, not only for the nutritional contributions but also because of its high content of starch, considered ideal for the industrial production of flours and starches. This research identified the structural and functional properties of flours and starches obtained from four varieties of sweet potato grown in the Colombian Caribbean region. The functional properties analyzed were the capacity of absorption of water, amylose, maximum viscosity, temperature of gelatinization, settlement, stability and structural behavior which was studied by FT-IR-infrared spectroscopy. Results presented variations in genotypes and localities evaluated, the viscoamilogram in flour showed encouraging values for the industry, both the maximum viscosity $(300 \mathrm{cP})$, the breakdown (cP -15.64) and setback $(186,65 \mathrm{cP})$, while starches showed relatively high values, therefore it is recommended to perform modification techniques to the native structure of the starch (physical, chemical or enzymatic modifications) to expand its applications. The structural behaviour showed signs indicating the presence of groups $\mathrm{OH}, \mathrm{CH}, \mathrm{CO}, \mathrm{H}_{2} \mathrm{O}$ in evaluated flours and sweet potato starches.

Keywords: genotype; flour; starch; viscoamillogram; spectroscopy.

\section{INTRODUCCIÓN}

La batata (Ipomea batatas Lam.) es una de las plantas bulbosas más cultivadas, a nivel mundial y se posiciona favorablemente como alimento saludable o "funcional", por su alto contenido de fibra dietética (3,1\%), proteínas (2,3\%), vitamina C (20-30mg/100g), potasio (200-300mg/100g), hierro (0,8mg/100g), calcio (11mg/100g) y compuestos antioxidantes, como ácidos fenólicos, antocianinas, tocoferoles y B-carotenos (Flórez et al. 2016). Es un alimento de alta energía y sus raíces poseen un contenido de carbohidratos totales de 25-30\%, de los cuales, el 98\% es considerado fácilmente digestible (Vargas \& Hernández, 2012).

El cultivo de la batata es muy importante, por las grandes posibilidades que tiene como componente de procesos industriales; la raíz de la batata, se ha integrado, esencialmente, a la industria alimenticia para producción de almidón, harina y una variedad de productos procesados, como dulces, productos de pastelería, industria de embutidos, entre otros (Flórez et al. 2016).

Es comercializada, principalmente, como producto en fresco ( $\mathrm{Li}$ et al. 2010) y como fuente de harina y de almidón, de alto valor energético, lo que convierte a la batata como la principal opción para sustituir las harinas consumidas en fresco, como la de papa y yuca (Vargas \& Hernández, 2012). A su vez, el almidón de batata, se ha considerado una fuente amilácea con un gran potencial, dado que el contenido de amilosa puede variar entre 15-25\% (Hernández et al. 2016). En este estudio, se evaluaron las propiedades funcionales y estructurales de cuatro variedades de batata, cultivadas en tres localidades de la región Caribe, buscando, a partir de los resultados, promover su siembra, como una opción agrícola real, en la que se le dé prioridad a la recuperación de este tubérculo, como un recurso genético, para la generación de alternativas viables y provechosas de la industria alimentaria y no alimentaria.

\section{MATERIALES Y MÉTODOS}

Materia prima. Se estudiaron tres variedades de batatas, que fueron genéticamente modificadas por la inserción de transgenes (plantas transgénicas) (Chinú, 440224, 199064-1) y una variedad nativa (criolla de la región Caribe), sembradas en la región Caribe colombiana, suministradas por la Corporación Colombiana de Investigación Agropecuaria (Corpoica). Previamente a la caracterización, se tomaron muestras, que fueron sometidas a deshidratación, a $60^{\circ} \mathrm{C}$, hasta humedad de 0 a 12\% y molienda, en un molino convencional, hasta conseguir una textura harinosa. La extracción de almidón, se realizó de forma manual, utilizando agua, según la metodología descrita por Pacheco \& Techeira (2008).

Caracterización funcional de las harinas y almidones. La capacidad de absorción de agua (CAA), se determinó por centrifugación, según la metodología descrita por la AACC (2012). Las propiedades de la pasta, se evaluaron con suspensiones al 4\% (w/v), en un reómetro (Anton Parr, MCR 302, Austria), usando el método descrito por Rivas (2012) y por Nasrin \& Anal (2014). Los resultados obtenidos, se procesaron con RheoCompass software, la versión 1.17 (Anton Paar, Austria).

Caracterización estructural de las harinas y almidones. Los espectros de infrarrojo fueron obtenidos con espectrofotómetro (Thermo scientific, IS50, USA), en un rango de $500-4000 \mathrm{~cm}^{-1}$; las muestras fueron mezcladas con sales de $\mathrm{KBr}$, a razón de 1:5, respectivamente (Colussi et al. 2015). Por su parte, el contenido de amilosa fue determinado solo a los almidones, con una leve modificación, precisando la absorbancia, en un espectrofotómetro, las UV- visible (Thermo Scientific Evolution 60 S, USA), a $620 \mathrm{~nm}$ (Salcedo et al. 2016).

Diseño experimental. Se empleó un diseño categórico multifactorial: el factor $\mathrm{A}$, que representa la localidad, con los siguientes niveles: L1 $=$ Corozal, L2=Cerete, L3=E1 Carmen de Bolívar y el factor B, las variedades de batata: V1= Chinú, V2= 440224, V3 = 199064-1 y V4= Criolla. Las variables estudiadas fueron CAA, propiedades de la pasta y espectros de infrarrojo de las harinas de batata; CAA, propiedades de la pasta, contenido de amilosa y espectros de infrarrojo de los almidones. Los datos obtenidos fueron analizados con el paquete estadístico Statgraphics, mediante un análisis de varianza, con un nivel de significancia del 5\% y para la comparación de medias, se utilizó la prueba de Tukey.

\section{RESULTADOS Y DISCUSIÓN}

La CAA de las harinas de batatas, que se muestra en la tabla 1, evidencia diferencias significativas entre las localidades, las variedades y entre la interacción de variedades y localidades $(p<0,05)$. Cereté y la variedad Chinú registraron los mayores valores, con un promedio de 290,9\%. Los resultados obtenidos coinciden con los 
expuestos por Salcedo et al. (2016), quienes reportaron un valor de CAA de $227 \%$, para harinas extraídas de diversas variedades de batata; La CAA es la capacidad de la harina para absorber el agua e hincharse, para mejorar la consistencia en los alimentos (Techeira et al. 2014; Sacón et al. 2016). Los altos valores de esta propiedad son ideales para la producción de harinas (Sacón et al. 2016), que se emplean como sustituto parcial de harinas de trigo en la elaboración de masa para panadería, debido a que se ha demostrado que, cuanta más agua absorba una harina, más cantidad de pan se podrá elaborar (Henao \& Aristizábal, 2009).
Sobre la CAA de los almidones influyeron significativamente las localidades, las variedades y la interacción de variedades y localidades $(\mathrm{p}<0,05)$. Las batatas cultivadas en la localidad del Carmen, con valores entre 88,7 y $96,3 \%$, registraron los mayores porcentajes de CAA, lo que se encuentra dentro de lo reportador por Das et al. (2010) y Pramodrao \& Riar (2014), con un rango de 77,38-89\% para almidones de batata y otros tubérculos, como la yuca; mantienen una CAA mayor que los almidones evaluados, siendo de $228 \%$, según Granados et al. (2014). Se ha demostrado que la CAA es

Tabla 1. Caracterización tecno-funcional de harinas y de almidones de batata.

\begin{tabular}{|c|c|c|c|c|}
\hline \multirow{2}{*}{ Parámetro } & Variedad & \multicolumn{3}{|c|}{ Localidad } \\
\cline { 2 - 5 } & & Corozal & Cereté & Carmen de Bolívar \\
\hline \multirow{4}{*}{$\begin{array}{c}\text { CAA harinas } \\
\text { (\%) }\end{array}$} & Chinú & $264,07 \pm 1,26^{\mathrm{Ba}}$ & $290,90 \pm 1,17^{\mathrm{Aa}}$ & $290,90 \pm 1,17^{\mathrm{Aa}}$ \\
\cline { 2 - 5 } & 440224 & $191,96 \pm 1,22^{\mathrm{Ac}}$ & $185,60 \pm 2,48^{\mathrm{Bd}}$ & $185,60 \pm 2,48^{\mathrm{Bd}}$ \\
\cline { 2 - 5 } & $199064-1$ & $175,05 \pm 1,47^{\mathrm{Bd}}$ & $192,83 \pm 0,70^{\mathrm{Ac}}$ & $192,83 \pm 0,70^{\mathrm{Ac}}$ \\
\hline \multirow{3}{*}{$\begin{array}{c}\text { CAA almidón } \\
(\mathbf{9} \%)\end{array}$} & Criolla & $218,47 \pm 1,79^{\mathrm{Ab}}$ & $215,69 \pm 1,36^{\mathrm{Ab}}$ & $215,69 \pm 1,36^{\mathrm{Ab}}$ \\
\cline { 2 - 5 } & Chinú & $73,21 \pm 1,04^{\mathrm{Bb}}$ & $75,22 \pm 1,03^{\mathrm{Cbc}}$ & $93,37 \pm 0,82^{\mathrm{Ab}}$ \\
\cline { 2 - 5 } & 440224 & $72,77 \pm 0,65^{\mathrm{Bb}}$ & $76,94 \pm 1,23^{\mathrm{Cc}}$ & $91,82 \pm 0,81^{\mathrm{Ac}}$ \\
\cline { 2 - 5 } & $199064-1$ & $74,69 \pm 0,89^{\mathrm{Ba}}$ & $68,02 \pm 0,63^{\mathrm{Ca}}$ & $96,32 \pm 0,60^{\mathrm{Ac}}$ \\
\hline
\end{tabular}

Medias con letras minúsculas diferentes en columnas y con letras mayúsculas, diferentes en filas indican diferencia estadísticamente significativa, conforme a la prueba de Tukey $(p<0,05)$.

mayor en almidones, en donde la amilosa y la amilopectina están apenas asociadas (Das et al. 2010). En este sentido, la diferencia de CAA de los almidones evaluados fue dependiente del contenido de amilosa, que arrojó los mayores valores para la localidad del Carmen de Bolívar y el testigo Criolla que, a su vez, fueron uno de los almidones que lograron la mayor absorción de agua.

Esta propiedad indica la capacidad de los productos alimenticios que contienen almidón para formar pastas o geles y permite determinar la textura y la calidad de los mismos, de modo que, los almidones que poseen una alta CAA, son destinados, principalmente, como aditivos alimentarios, dada su contribución a la textura en los sistemas alimentarios, siendo su utilización como agente espesante, su aplicación alimentaría más importante (Contreras et al. 2015).

Los parámetros evaluados para caracterizar las propiedades de la pasta de las harinas y los almidones de batata son la viscosidad máxima, la temperatura de gelatinización y los procesos de breakdown y setback. La figura 1 muestra el comportamiento de la viscosidad en suspensiones de harinas y de almidones de batata de las diferentes variedades y localidades, durante ciclos de calentamientoenfriamiento, que se resumen en la tabla 2.
La viscosidad máxima $(\mathrm{Vm})$ de las harinas evaluadas presentó diferencias significativas $(p<0,05)$ entre variedades y entre la interacción de variedades y localidades. El municipio de Cereté y la 199064-1 arrojaron los mayores promedios, de 397,59cP y 460,65Cp, respectivamente, mientras que Corozal y variedad Chinú presentaron los promedios más bajos (224,97cP y 110,57Cp, respectivamente). Aquellas harinas que presentaron mayor aumento en la viscosidad podrían ser empleadas en la elaboración de productos que requiera espesamiento en procesos de cocción, como mezclas de sopas y de pudines instantáneos (Lucas et al. 2013). La disminución en los valores de $\mathrm{Vm}$, se explica por la degradación por fricción de las moléculas de amilosa y amilopectina, que disminuyen su capacidad de retención de agua. Los valores de viscosidad máxima de las batatas evaluadas fueron inferiores a los reportados por Julianti et al. (2015), quienes obtuvieron un promedio de 1831Cp, para harina de batata y superior a los registrados por Aprianita et al. (2013), de 41,2Cp, para harina de batata; $31 \mathrm{Cp}$, para harina de ñame y 20,21Cp, para harina de yuca.

La Vm de los almidones evaluados osciló entre 2200 y $3100 \mathrm{cP}$, arrojando diferencias significativas entre las localidades y la interacción de variedades y localidades $(\mathrm{p}<0,05)$, obteniendo, para 

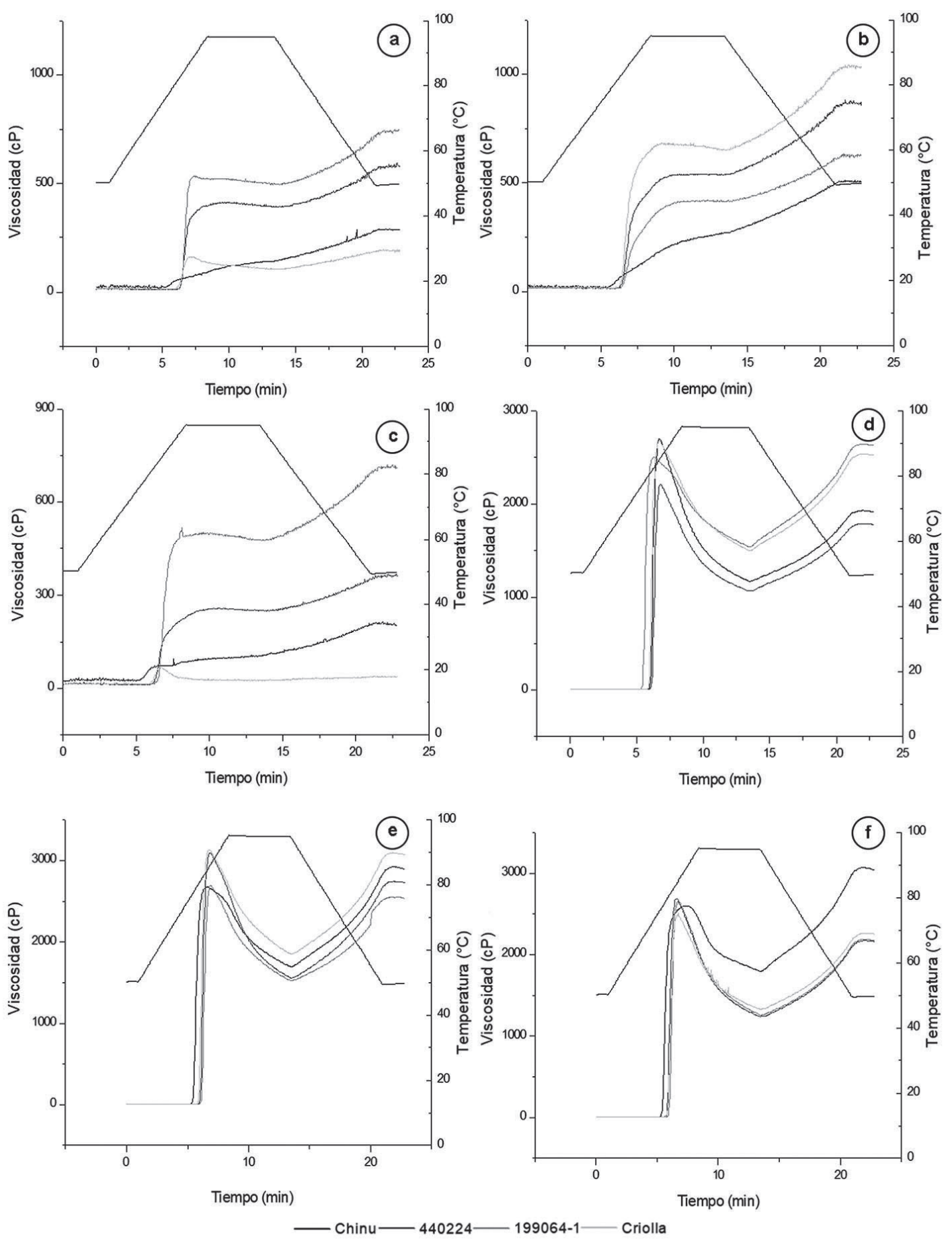

Figura 1. Viscoamilogramas de harinas y de almidones de batatas en las diferentes localidades. Harinas: a. Corozal; b. Cereté y c. Carmen. Almidones: d. Corozal; e. Cereté y f. Carmen 
Tabla 2. Comportamiento de la viscosidad en suspensión de batatas y almidones de batata durante ciclos de calentamiento-enfriamiento.

\begin{tabular}{|c|c|c|c|c|c|}
\hline \multirow[b]{2}{*}{ Batata } & \multirow[b]{2}{*}{ Propiedades } & \multirow[b]{2}{*}{ Variedad } & \multicolumn{3}{|c|}{ Localidad } \\
\hline & & & Corozal & Cereté & $\begin{array}{l}\text { Carmen } \\
\text { de Bolívar }\end{array}$ \\
\hline \multirow{16}{*}{ Harina } & \multirow{4}{*}{$\begin{array}{l}\text { Viscosidad } \\
\text { máxima (cP) }\end{array}$} & Chinú & $92 \pm 10,73^{\mathrm{Ab}}$ & $153 \pm 31,60^{\mathrm{Ac}}$ & $86 \pm 1,12^{\mathrm{Ab}}$ \\
\hline & & 440224 & $401 \pm 77,20^{\text {Aba }}$ & $465 \pm 78,30^{\text {Aab }}$ & $227 \pm 14,90^{\mathrm{Bb}}$ \\
\hline & & 199064-1 & $537 \pm 40,15^{\mathrm{Aa}}$ & $327 \pm 55,85^{\mathrm{Bbc}}$ & $516 \pm 75,45^{\mathrm{ABa}}$ \\
\hline & & criolla & $164 \pm 23,45^{\mathrm{Bb}}$ & $644 \pm 61,00^{\mathrm{Aa}}$ & $68 \pm 10,14^{\mathrm{Bb}}$ \\
\hline & \multirow{4}{*}{$\begin{array}{c}\text { Temperatura de } \\
\text { gelatinización } \\
\left({ }^{\circ} \mathrm{C}\right)\end{array}$} & Chinú & $74,30 \pm 0,10^{\mathrm{Bb}}$ & $77,83 \pm 0,15^{\mathrm{Ab}}$ & $79,60 \pm 0,10^{A a}$ \\
\hline & & 440224 & $79,70 \pm 0,20^{\mathrm{Aa}}$ & $80,400 \pm 0,10^{\mathrm{Aa}}$ & $79,40 \pm 0,20^{\text {Aa }}$ \\
\hline & & $199064-1$ & $76,07 \pm 1,97^{\mathrm{Bb}}$ & $78,90 \mathrm{ui} \pm 1,04^{\mathrm{Aab}}$ & $79,60 \pm 0,20^{\mathrm{Aa}}$ \\
\hline & & criolla & $79,47 \pm 0,25^{\mathrm{Aa}}$ & $79,43 \pm 0,25^{\text {Aab }}$ & $79,50 \pm 0,10^{\mathrm{Aa}}$ \\
\hline & \multirow{4}{*}{ Breakdown (cP) } & Chinú & $-50,2 \pm 15,53^{\mathrm{Ab}}$ & $-113,8 \pm 15,00^{\mathrm{Bb}}$ & $-17,6 \pm 6,46^{\mathrm{Ab}}$ \\
\hline & & 440224 & $7,8 \pm 29,60^{\mathrm{Aa}}$ & $-71,1 \pm 13,05^{\mathrm{Bb}}$ & $-23,6 \pm 0,15^{\mathrm{ABb}}$ \\
\hline & & 199064-1 & $38,6 \pm 7,20^{\mathrm{Aa}}$ & $-87,9 \pm 16,90^{\mathrm{Bb}}$ & $39,3 \pm 0,80^{\mathrm{Aa}}$ \\
\hline & & criolla & $56,5 \pm 10,29^{\mathrm{Aa}}$ & $-8,7 \pm 61,40^{\mathrm{Ba}}$ & $43,1 \pm 8,34^{\mathrm{Aa}}$ \\
\hline & \multirow{4}{*}{ Setback (cP) } & Chinú & $141,9 \pm 70,10^{\text {Abab }}$ & $262,9 \pm 74,99^{\mathrm{Aab}}$ & $98,3 \pm 11,96^{\mathrm{Bab}}$ \\
\hline & & 440224 & $170,8 \pm 61,40^{\text {Abab }}$ & $305,2 \pm 77,20^{\text {Aab }}$ & $110,7 \pm 9,10^{\mathrm{Bab}}$ \\
\hline & & 199064-1 & $241,3 \pm 16,77^{\mathrm{Aa}}$ & $210,8 \pm 7,96^{\mathrm{Ab}}$ & $229,9 \pm 32,20^{\mathrm{Aa}}$ \\
\hline & & criolla & $87,2 \pm 19,21^{\mathrm{Bb}}$ & $369,1 \pm 84,16^{\mathrm{Aa}}$ & $11,5 \pm 0,69^{\mathrm{Bb}}$ \\
\hline \multirow{16}{*}{ Almidón } & \multirow{4}{*}{$\begin{array}{l}\text { Viscosidad } \\
\text { máxima (cP) }\end{array}$} & Chinú & $2703 \pm 22,74^{\mathrm{Aa}}$ & $2751 \pm 5,76^{\mathrm{Aa}}$ & $2601 \pm 11,00^{\mathrm{Aa}}$ \\
\hline & & 440224 & $2211 \pm 1,54^{\mathrm{Bb}}$ & $3095 \pm 2,03^{\mathrm{Aa}}$ & $2689 \pm 14,30^{\mathrm{Aa}}$ \\
\hline & & 199064-1 & $2410 \pm 5,54^{\text {Aab }}$ & $2694 \pm 17,01^{\mathrm{Aa}}$ & $2671 \pm 8,19^{A a}$ \\
\hline & & criolla & $2677 \pm 12,14^{\mathrm{Bab}}$ & $3134 \pm 5,59^{\mathrm{Aa}}$ & $2472 \pm 5,59^{\mathrm{Ba}}$ \\
\hline & \multirow{4}{*}{$\begin{array}{c}\text { Temperatura de } \\
\text { gelatinización } \\
\left({ }^{\circ} \mathrm{C}\right)\end{array}$} & Chinú & $80,1 \pm 0,00^{\mathrm{Aa}}$ & $74,533 \pm 0,65^{\mathrm{Bb}}$ & $73,4 \pm 0,50^{\mathrm{Cc}}$ \\
\hline & & 440224 & $79,433 \pm 0,15^{\mathrm{Bb}}$ & $80,133 \pm 0,06^{\mathrm{Aa}}$ & $78,833 \pm 0,12^{\mathrm{Cb}}$ \\
\hline & & 199064-1 & $74,3 \pm 0,00^{\mathrm{Bc}}$ & $79,733 \pm 0,12^{\mathrm{Aa}}$ & $79,933 \pm 0,12^{\mathrm{A}_{2}}$ \\
\hline & & criolla & $80 \pm 0,00^{\mathrm{Aa}}$ & $79,733 \pm 0,06^{\mathrm{Aa}}$ & $79,67 \pm 0,06^{\mathrm{Aa}}$ \\
\hline & \multirow{4}{*}{ Breakdown (cP) } & Chinú & $1526 \pm 25,36^{\mathrm{Aa}}$ & $1045 \pm 78,06^{\mathrm{Bb}}$ & $801 \pm 28,62^{\mathrm{Bb}}$ \\
\hline & & 440224 & $1137 \pm 11,85^{\mathrm{Bb}}$ & $1527 \pm 41,53^{\mathrm{Aa}}$ & $1445 \pm 20,60^{\mathrm{Aba}}$ \\
\hline & & 199064-1 & $867 \pm 77,02^{\mathrm{Bb}}$ & $1169 \pm 29,14^{\mathrm{ABab}}$ & $1401 \pm 36,61^{\mathrm{Aa}}$ \\
\hline & & criolla & $1169 \pm 9,85^{\text {Aab }}$ & $1272 \pm 59,00^{\text {Aab }}$ & $1148 \pm 77,97^{\mathrm{Aab}}$ \\
\hline & \multirow{4}{*}{ Setback (cP) } & Chinú & $720 \pm 3,65^{\mathrm{Bb}}$ & $1189 \pm 1,85^{\text {Bab }}$ & $1239 \pm 4,22^{\mathrm{Ba}}$ \\
\hline & & 440224 & $698 \pm 7,51^{\mathrm{Bb}}$ & $1155 \pm 2,19^{\mathrm{Bbc}}$ & $919 \pm 6,08^{\mathrm{Bb}}$ \\
\hline & & 199064-1 & $1037 \pm 9,25^{\mathrm{Ba}}$ & $908 \pm 12,53^{\mathrm{Bc}}$ & $904 \pm 6,65^{\mathrm{Bb}}$ \\
\hline & & criolla & $1014 \pm 13,20^{\mathrm{Ba}}$ & $1204 \pm 4,47^{\mathrm{Ba}}$ & $912 \pm 5,87^{\mathrm{Bb}}$ \\
\hline
\end{tabular}

Medias con letras minúsculas diferentes en columnas y con letras mayúsculas diferentes en filas, indican diferencia estadísticamente significativa, conforme a la prueba de Tukey $(\mathrm{p}<0,05)$.

Corozal y para el genotipo 199064-1, los menores valores y para Cereté y el testigo Criolla, las Vm más elevadas, durante el proceso de pastificación. Este comportamiento, posiblemente, se deba a que los gránulos de dichos almidones tienen mayor tendencia a absorber agua en el proceso de calentamiento y presentan mayor tamaño, lo que los lleva a ocupar un mayor volumen y otorgar una mayor viscosidad. Los valores de viscosidad máxima de los almidones evaluados fueron consistentes con estudios realizados por Zhou et al. (2014), quienes reportaron un valor de 2109Cp, para almidón de batatas transgénicas; por Barragán et al. (2016), con 2470Cp, para almidón nativo de yuca y por Paternina et al. (2016), con un rango de 1900 a 3200Cp, para almidones de yuca, ñame y batata. 
Los almidones que presentaron alta viscosidad de pasta durante el calentamiento podrían ser excelentes espesantes para la elaboración de alimentos para niños y para la industria de confitería (Salcedo $e t$ al. 2016) y se sugiere su posible utilización en la elaboración de otros productos, como salsas sometidas a calentamiento, aderezos para ensalada, sopas instantáneas, colados y pudines, entre otros. Por su parte, almidones de batata con bajas viscosidades son beneficiosos para algunas aplicaciones alimenticias, incluyendo dulces y gomas, para lo cual, se recomendaría implementar procesos de modificación, que permitan mejorar las propiedades funcionales de los almidones de batata, como es el caso de reducir su viscosidad y conseguir una mayor estabilidad a los procesos de calentamiento y enfriamiento (Aprianita et al. 2013).

Sobre la temperatura de gelatinización de las harinas y almidones evaluados influyó, significativamente, la localidad, la variedad y la interacción de variedades y localidades. En las harinas, los mayores valores fueron registrados para el Carmen de Bolívar y el genotipo 440224 (79,5 y $79,8^{\circ} \mathrm{C}$, respectivamente). Las temperaturas de gelatinización ( $\mathrm{Tg}$ ) obtenidas para las harinas en estudio fueron similares a las reportadas por Aprianita et al. (2013), para harina de batata, ñame y yuca.

Por otra parte, la $\mathrm{Tg}$ en los almidones evaluados de la variedad testigo criolla, con un valor de $79,80^{\circ} \mathrm{C}$ y la localidad del Cereté, con $78,53^{\circ} \mathrm{C}$, registraron los mayores promedios. Los valores registrados para la temperatura de gelatinización de los almidones evaluados fueron consistentes con los reportados en estudios previos, donde la temperatura de gelatinización del almidón nativo de batata oscila desde 74cP (Charles et al. 2015) hasta 80cP (Zhou et al. 2014). A su vez, se ha demostrado que otras fuentes tuberculosas, como el ñame, con $66,8^{\circ} \mathrm{C}$ y la yuca, de $61,4^{\circ} \mathrm{C}$, reportada por Aprianita et al. (2013), presentan una menor temperatura de gelatinización.

La $\operatorname{Tg}$ es una característica importante, que se debe considerar al momento de desarrollar un producto alimenticio, ya que las harinas y los almidones de batata que mostraron los mayores valores de $\mathrm{Tg}$, requerirán mayor temperatura, para iniciar el proceso de absorción de agua, hinchamiento y gelificación y necesitarían de un mayor tiempo de cocción (Paternina et al. 2016). Por su parte, la baja temperatura de gelatinización proporciona una mayor disponibilidad de almidón a las enzimas amilolíticas durante el proceso de cocción, que es deseable en la fabricación de pan (Julianti et al. 2015).

Se observaron diferencias significativas entre la máxima viscosidad y la viscosidad alcanzada después del periodo de calentamiento $(\mathrm{p}<0,05)$, evidenciando que las localidades y las variedades de batata ejercieron un efecto sobre la estabilidad de las harinas evaluadas, donde la variedad Chinú, cultivada en el municipio de Cereté, arrojó la menor estabilidad, con un valor cerca de $-113,8 \mathrm{cP}$, mientras que la 440224, en Corozal, obtuvo un valor de apenas $7,8 \mathrm{cP}$, presentando, así, la mayor estabilidad ante procesos de calentamiento, en comparación con las demás variedades. Los resultados obtenidos son inferiores a los registrados por Julianti et al. (2015), con un valor de $829 \mathrm{cP}$, para harinas de batata; a su vez, algunas de las variedades de batatas evaluadas mantuvieron una tendencia similar a la reportada por Aprianita et al. (2013), con un breakdown de $17,9 \mathrm{cP}$, para harinas de batata y para harinas extraídas de otros tubérculos y raíces, como el ñame, con 12,9cP y yuca, con $8,9 \mathrm{cP}$.

En este sentido, en aquellas suspensiones de harinas, donde se presentaron los mayores valores de breakdown, posiblemente, predomina una estructura intragranular muy poco resistente, con una mayor cantidad de enlaces débiles y un menor grado de cristalinidad y, por tanto, gránulos muy frágiles, que se destruyen fácilmente, por efecto del calentamiento, solubilizándose muy rápido; mientras que, con valores negativos de breakdown, se puede suponer que los gránulos hinchados son frágiles y no resisten el proceso de agitación y calentamiento. En cambio, valores cercanos a cero en estabilidad, indican la presencia de gránulos de harina que son más resistentes al estrés térmico y mecánico y se destruyen con menor facilidad. Por lo tanto, los gránulos de harinas de las batatas de la variedad 440224, cultivadas en Corozal, son más resistentes al esfuerzo mecánico, en comparación con las demás; esta propiedad es crucial para la formulación de productos alimenticios que requieran mantener su viscosidad estable durante períodos de calentamiento prolongado y en condiciones de agitación mecánica constante (Lee et al. 2012; Paternina et al. 2016).

Las variedades y la interacción de localidades y las variedades ejercieron un efecto sobre la estabilidad de los almidones, lo cual, indica que dichos almidones presentan diferentes susceptibilidades a la degradación, causada por calentamiento bajo estrés mecánico, como ocurre en la cocción (Salcedo et al. 2016). La variedad 440224, cultivada en la localidad de Cereté, presentó una menor resistencia a este tipo de procesos mecánicos, mientras que la variedad 1990641 ofrece la mayor resistencia. En términos generales, los valores obtenidos indican que los almidones nativos de batata no son resistentes al estrés mecánico y térmico y, por ende, presentan menor estabilidad, que puede estar asociada a la presencia de una estructura intragranular más débil, lo que origina que sean más frágiles y susceptibles a la ruptura, por fuerzas térmicas y mecánicas (Paternina et al. 2016).

La variedad criolla, que es la nativa de la región, por ser la que presentó mayor viscosidad, una temperatura de gelatinización relativamente alta y buena estabilidad con respecto a las demás variedades, puede ser utilizada en la preparación de alimentos que requieren altas temperaturas, que sean resistentes al calor y que produzcan alta viscosidad, como confitería dura y en la elaboración de alimentos para niños (Salcedo et al. 2016).

Para las harinas de las batatas en estudio, el setback o retrogradación, arrojó diferencias significativas $(p<0,05)$, entre localidad y entre la interacción de variedades y localidades. La localidad de Cereté y la variedad $199064-1$ presentaron los mayores valores $(287,03 \mathrm{cP}$ y $227,35 \mathrm{cP}$, respectivamente), mientras que para el Carmen de Bolívar y el genotipo Criolla registraron los valores más bajos (112,62cP y $155,95 \mathrm{cP}$, respectivamente). Los resultados obtenidos son inferiores a lo demostrado por Julianti et al. (2015), con un valor de 563cP, en harina de batata y $1311 \mathrm{cP}$, para harina de trigo y, superior, a lo 
reportado por Aprianita et al. (2013), en harina de ñame, con 10,3cP, en harina de batata, con $7,4 \mathrm{cP}$ y en harina de yuca, con $5,4 \mathrm{cP}$. Las harinas de batata con respecto al índice de retrogradación pueden estar relacionadas con el contenido de almidón, que representa mayor tendencia a la retrogradación y, por tanto, menos estable serán a procesos de enfriamiento (Lucas et al. 2013). Esto por la retrogradación de los gránulos de almidón (reorganización de las moléculas y convirtiéndose en una estructura de gel) (García et al. 2012).

Por su parte, como se muestra en la tabla 2, las variedades, las localidades y la interacción entre los genotipos de batata y las condiciones de la localidad en que se cultivaron influyeron significativamente en los valores de retrogradación de los almidones evaluados $(p<0,05)$. Las batatas cultivadas en la localidad de Cereté y de la variedad Chinú obtuvieron una mayor tendencia a la retrogradación o una menor resistencia a la misma, registrando, así, los mayores valores, en promedio de 1129,7 y $1056 \mathrm{cP}$, respectivamente, mientras que para Corozal y el genotipo 440224, se obtuvieron los valores más bajos (alrededor de 883,7 y $924 \mathrm{cP}$, respectivamente). Los resultados obtenidos son consistentes con valores de referencia reportados por Zhou et al. (2014), quien plantean que la viscosidad de retrogradación oscila entre 911-1839cP, para almidones de batatas transgénicas; por Barragán et al. (2016), con un valor alrededor de 966cP, para almidón nativo de yuca y para otros tubérculos y raíces, de 900 a 2000cP (Paternina et al. 2016).

Como se observa en la figura 1, durante el ciclo de enfriamiento, la viscosidad final de todas las pastas de almidón aumentó rápidamente, debido a la formación de un gran número de enlaces intermoleculares de hidrógeno, lo que conduce a la formación de gel a baja temperatura. La viscosidad final indica la capacidad del almidón para formar una pasta después del enfriamiento y el aumento de la estabilidad de la pasta se acompaña con bajos valores de ruptura; la viscosidad final, el aumento secundario durante la fase de enfriamiento, se asocia con el fenómeno de retrogradación (Mishra \& Rai, 2006). Los almidones de las batatas cultivadas en la localidad de Cereté y la variedad Chinú arrojaron los mayores incrementos en la viscosidad, cuando la pasta fue enfriada, lo que indica que no es estable a los procesos de enfriamiento; esto se debe tener en cuenta al momento de pensar en incorporar el almidón a algún producto que necesite enfriamiento durante su preparación y se recomienda su empleo, como aditivos en la industria de confitería en el desarrollo de caramelos (Figueroa et al. 2016). Por su parte, los almidones de batata cultivados en Corozal y de la variedad 199064-1, con una mayor estabilidad a los procesos de enfriamiento, podrían ser usados en alimentos congelados o que necesiten enfriamiento durante su preparación, como los flanes y pudines (Aprianita et al. 2013; Paternina et al. 2016); no obstante, en términos generales, los almidones de batata evaluados mostraron una alta tendencia hacia la retrogradación, por lo que es necesario implementar estrategias de modificación, para ampliar sus aplicaciones.

Los FT-IR (Figura 2), tanto de las harinas como de los almidones de batata, mostraron un comportamiento similar en las señales de absorbancia, desde 400 hasta $4000 \mathrm{~cm}^{-1}$. Por su parte, las harinas de batata registraron algunas bandas de absorción, características de la estructura del almidón y la presencia de otros materiales. El intervalo de absorción, entre 3381 y $3363 \mathrm{~cm}^{-1}$, corresponden al alargamiento vibracional de los grupos de los enlaces $\mathrm{OH}$, lo que indica una importante contribución de las moléculas de agua (Yaacob et al. 2011). El segundo pico observado oscila alrededor de los $2928 \mathrm{~cm}^{-1}$ y se atribuye al estiramiento de los enlaces $\mathrm{CH}$ (asimétrico $\mathrm{CH}_{2}$ ) (Tapia et al. 2010). La absorción débil entre $1.636-1.643 \mathrm{~cm}^{-1}$ es debida al estiramiento vibracional de los enlaces $\mathrm{CO}$ e identifica el compuesto amida I. Otra banda importante concerniente a las proteínas es la relativa a la amida III, que absorbe entre $1.200-1.350 \mathrm{~cm}^{-1}$ y surge del estiramiento y doblado del enlace $\mathrm{CN}$ del grupo amida de las proteínas. Asimismo, se presentaron bandas alrededor de $1.410 \mathrm{~cm}^{-1}$, debido a la deformación asimétrica del enlace $\mathrm{CH}_{3}$ (Rojas et al. 2016). Finalmente, la señal encontrada en el intervalo de 1.240$990 \mathrm{~cm}^{-1}$ son características del estiramiento CO del grupo éster en la estructura $\mathrm{COC}$ y en $\mathrm{COH}$ del anillo glicosídico de los gránulos de almidón, presentes en la harina (Flores et al. 2014), identificando las vibraciones típicas de los hidratos de carbono.

El comportamiento registrado por los almidones presentó un intervalo de absorción de una banda ancha y fuerte entre 3.385 y $3.423 \mathrm{~cm}^{-1}$; estas bandas corresponden a alargamientos de los $\mathrm{OH}$ de los grupos hidroxilo libres enlazados intra e intermolecularmente, presentes en las unidades de anhidroglucosa de la amilosa y la amilopectina (Guo et al. 2014). Longitudes de onda entre 2.850 $3.000 \mathrm{~cm}^{-1}$, se asocian a la tensión CH de la unidad de anhidra glucosa de las moléculas de almidón, indicando la presencia de grupos metilenos $\left(\mathrm{CH}_{2}\right)$ (Enríquez et al. 2013); los cambios de intensidad en este rango, se pueden atribuir a las variaciones en la cantidad de amilosa y amilopectina presentes en los almidones (Andrade $e t$ al. 2012). Los picos cercanos a $990 \mathrm{~cm}^{-1}$, se asocian al enlace CO de COC (Guo et al. 2014). El comportamiento observado para los espectros FT-IR evaluados (Figura 2) coinciden con la identificación molecular de almidones nativos, expuesta por Salcedo et al. (2016), en el cual, se analiza cualitativa y cuantitativamente almidones de diferentes cultivares de batata, presentándose los picos más relevantes, en una franja comprendida entre 3.900 y $1.087 \mathrm{~cm}^{-1}$, indicando la presencia de grupos $\mathrm{OH}, \mathrm{CH}$ y $\mathrm{CO}$; a su vez, mantuvo similitud con almidones nativos de yuca y ñame, donde se muestran rangos de longitud de onda entre 500 y $4.000 \mathrm{~cm}^{-1}$ y picos característicos en $3.000-3.900 \mathrm{~cm}^{-1}$ y $1.087 \mathrm{~cm}^{-1}$ (Figueroa et al. 2016; Salcedo et al. 2016).

En la tabla 3, el porcentaje de amilosa de los almidones de batata evidencian que existen diferencias significativas entre las localidades, las variedades y la interacción de variedades y localidades $(p<0,05)$. El Carmen de Bolívar, la variedad Chinú y el testigoCriolla presentaron los mayores valores (alrededor de 23\%). Los valores obtenidos coincidieron con Tecson (2007), quien reportó un rango de 12,90 a $29,70 \%$, para batata y, para almidones de otros tubérculos, como el ñame, con 22,66\% (Figueroa et al. 2016). Los almidones ricos en amilosa son preferidos comercialmente, por los geles que se adquieren con ellos; presentan mejores propiedades mecánicas, son menos solubles y muestran mayor resistencia a la degradación química o enzimática. Industrialmente, el rango amilosa/amilopectina, se puede manipular genética, física y 

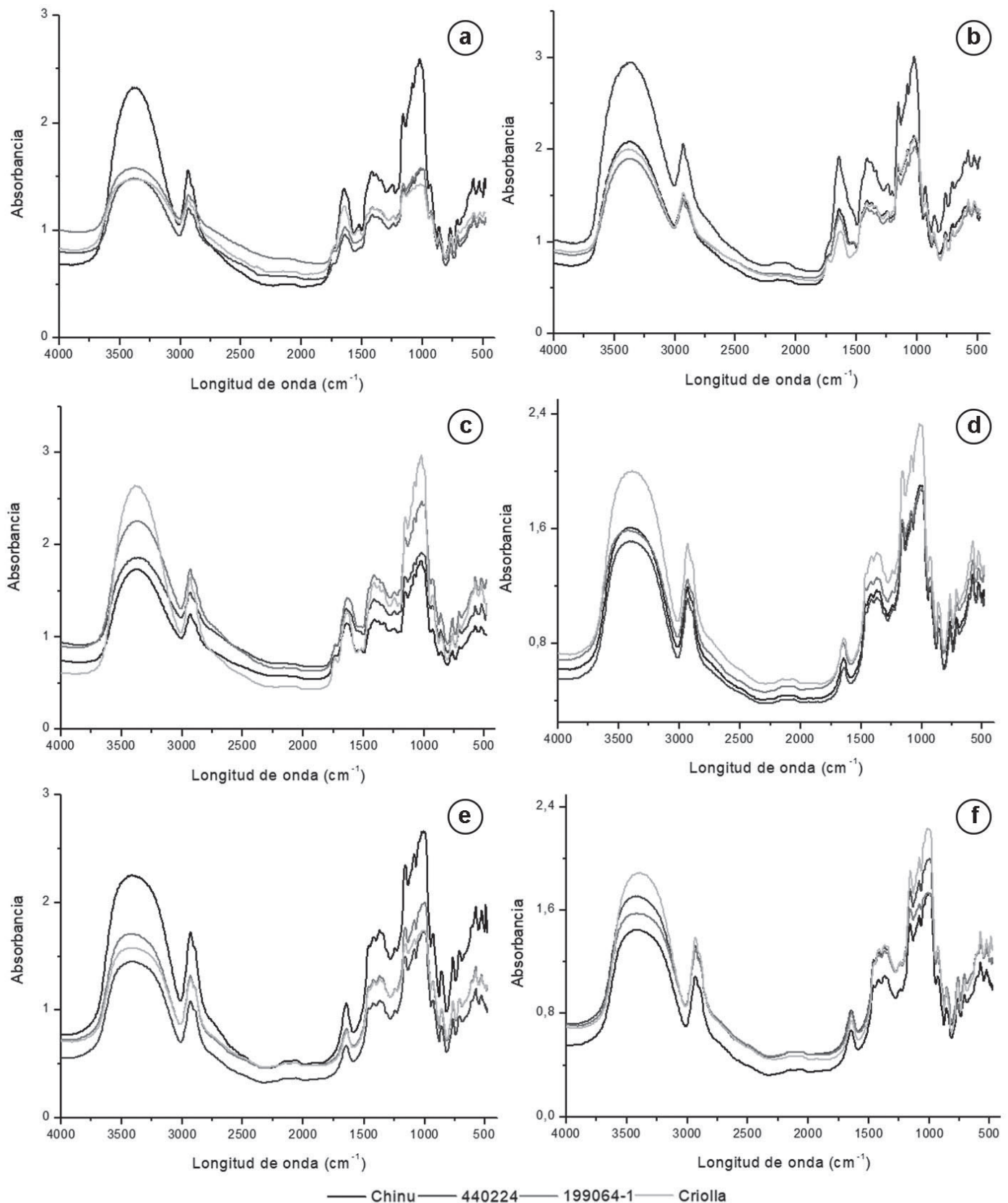

Figura 2. FT-IR para harinas y para almidones de batatas, en las diferentes localidades. Harinas: a. Corozal; b. Cereté y c. Carmen. Almidones: d. Corozal; e. Cereté y f. Carmen.

Tabla 3. Amilosa de los almidones de batata.

\begin{tabular}{|c|c|c|c|c|}
\hline \multirow{2}{*}{ Parámetro } & \multirow{2}{*}{ Variedad } & \multicolumn{3}{|c|}{ Localidad } \\
\cline { 3 - 5 } & & Corozal & Cerete & Carmen de Bolívar \\
\hline \multirow{3}{*}{$\begin{array}{c}\text { Amilosa en el } \\
\text { almidón (\%) }\end{array}$} & Chinú & $21,96 \pm 0,09^{\mathrm{Ba}}$ & $21,33 \pm 0,08^{\mathrm{Ca}}$ & $23,56 \pm 0,02^{\mathrm{Ac}}$ \\
\cline { 2 - 5 } & 440224 & $17,12 \pm 0,05^{\mathrm{Cd}}$ & $20,45 \pm 0,09^{\mathrm{Bc}}$ & $24,88 \pm 0,05^{\mathrm{Aa}}$ \\
\cline { 2 - 5 } & $199064-1$ & $19,29 \pm 0,23^{\mathrm{Cc}}$ & $20,92 \pm 0,02^{\mathrm{Bb}}$ & $22,56 \pm 0,11^{\mathrm{Ad}}$ \\
\cline { 2 - 5 } & Criolla & $21,26 \pm 0,03^{\mathrm{Bb}}$ & $20,31 \pm 0,02^{\mathrm{Cc}}$ & $24,53 \pm 0,11^{\mathrm{Ab}}$ \\
\hline
\end{tabular}

Medias con letras minúsculas diferentes en columnas y con letras mayúsculas diferentes en filas, indican diferencia estadísticamente significativa, conforme a la prueba de Tukey $(\mathrm{p}<0,05)$. 
químicamente, para modificar las características propias, como viscosidad, gelatinización, textura, solubilidad, estabilidad del gel y retrogradación, para darle propiedades industriales estables (Vargas \& Hernández, 2012).

La determinación del contenido de amilosa es realmente importante, ya que permite predecir el comportamiento reológico del almidón, determinar las condiciones de procesamiento más idóneas y evaluar la calidad de diferentes productos alimenticios. La fracción de amilosa imparte características definitivas al almidón y, por lo tanto, su concentración es considerada un criterio de calidad importante (Techeira et al. 2014).

En general, se puede concluir que las harinas de las batatas cultivadas en la localidad de Corozal mostraron baja capacidad para absorber agua, lo cual, condujo a que se presentaran los menores valores de viscosidad máxima que, junto a una baja temperatura de gelatinización y una buena estabilidad en procesos de calentamiento, es crucial para la elaboración de productos alimenticios, que requieran mantener una viscosidad estable. El almidón extraído de la variedad criolla, que es la nativa de la región, al presentar una buena estabilidad, una alta viscosidad máxima y una temperatura de gelatinización relativamente alta, presenta un gran potencial hacia la preparación de alimentos resistentes al calor y que produzcan alta viscosidad, como confitería dura y en la elaboración de alimentos para niños. Finalmente, teniendo en cuenta los valores de setback en las harinas, se pueden considerar como posible potencial para elaboración de productos de panadería, mientras que los almidones, por su alto contenido en amilosa, presentaron una tendencia hacia la retrogradación, por lo que es necesario implementar estrategias de modificación para ampliar sus aplicaciones.

Agradecimientos: Los autores agradecen al Grupo de investigación en Procesos Agroindustriales y Desarrollo Sostenible (PADES) de la Universidad de Sucre, por su apoyo, por permitir el desarrollo de esta investigación en sus instalaciones y a la Corporación Colombiana de Investigación Agropecuaria (CORPOICA), por las batatas suministradas. Conflicto de intereses: El manuscrito fue ejecutado, preparado y revisado con la participación de todos los autores, quienes declaramos que no existe conflicto de intereses que ponga en riesgo la validez de los resultados presentados. Financiación: Esta investigación fue financiada por el Grupo de investigación en Procesos Agroindustriales y Desarrollo Sostenible (PADES) e Ing. Químico. PhD. Jairo Salcedo Mendoza, en el 2017.

\section{REFERENCIAS}

1. AMERICAN ASSOCIATION OF CEREAL CHEMISTSAACC. 2012. Approved methods. 11 ed. Saint Paul, Minnesota: AACC.

2. ANDRADE, M.; TAPIA, D.; MENEGALLI, F. 2012. Physical-chemical, thermal, and functional properties of achira (Canna indica L.) flour and starch from different geographical origin. Starch-Stärke. 64(5):348-358. https:/ / doi.org/10.1002/star.201100149
3. APRIANITA, A.; VASILJEVIC, T.; BANNIKOVA, A.; KASAPIS, S. 2013. Physicochemical properties of flours and starches derived from traditional Indonesian tubers and roots. Journal of food science and technology. 51(12):36693679. https://doi.org/10.1007/s13197-012-0915-5

4. ASSOCIATION OF OFFICIAL ANALY'TICAL CHEMISTSAOAC. 2016. Official Methods of Analysis. 20th ed. Washington D.C: Association of Official Analytical Chemists. Cap 4. Page 1-77.

5. BARRAGÁN, K.; SALCEDO, J.; FIGUEROA, J. 2016. Propiedades tecno-funcionales de almidón modificado de yuca (Manibot esculenta C.) por pregelatinización tipo batch. Agronomía Colombiana. 34(1Supl):S317-S320. https://doi. org/10.15446/agron.colomb.sup.2016n1.58034

6. CHARLES, A.; CATO, K.; HUANG, T.; CHANG, Y.; CIOU, J.; CHANG, J.; LIN, H. 2015. Functional properties of arrowroot starch in cassava and sweet potato composite starches. Food Hydrocolloids. 53:187-191. https://doi. org/10.1016/j.foodhyd.2015.01.024

7. COLUSSI, R.; MELLO, S.; ZANELLA, V.; BARTZ, J.; GUTKOSKI, L.; ZAVAREZE, E.; GUERRA, A. 2015. Acetylation of rice starch in an aqueous medium for use in food. LWT-Food Science and Technology. 62(2):10761082. https://doi.org/10.1016/j.lwt.2015.01.053

8. CONTRERAS, M.; MARTÍNEZ, G.; ZAVALETA, M.; MACCHA, V. 2015. Gelatinización y gelificación de almidones. Universidad Nacional de Callao, Facultad de ingeniería química. 5(2):16-21.

9. DAS, A.; SINGH, G.; SINGH, S.; RIAR, C. 2010. Effect of acetylation and dual modification on physico-chemical, rheological and morphological characteristics of sweet potato (Ipomoea batatas) starch. Carbohydrate Polymers. 80(3):725732. https://doi.org/10.1016/j.carbpol.2009.12.018

10. ENRÍQUEZ, M.; VELASCO, R.; FERNÁNDEZ, A. 2013. Caracterización de almidones de yuca nativos y modificados para la elaboración de empaques biodegradables. Biotecnología en el Sector Agropecuario y Agroindustrial. 11(2):21-30.

11. FIGUEROA, J.; SALCEDO, J.; RODRÍGUEZ, M. 2016. Almidones acetilados de yuca y ñame para uso alimentario. Agronomía Colombiana. 34(1):S785-S788. https://doi. org/10.15446/agron.colomb.sup.2016n1.58002

12. FLÓREZ, D.; CONTRERAS, C.; URIBE, C. 2016. Perspectivas tecnológicas y comerciales para el cultivo de la batata en Colombia. Corporación Colombiana de Investigación Agropecuaria-Corpoica. Mosquera, Colombia. 110p. Disponible desde internet en: https:// repository.agrosavia. 
co/bitstream/handle/20.500.12324/13141/80391_67007. pdf?sequence $=1$ \&isAllowed $=\mathrm{y}($ con acceso el 06/06/2018).

13. FLORES, F.; LOZANO, F.; RAMOS, A.; SALGADO, R.; GUERRERO, V.; RAMÍREZ, S.; BELLO, L.; ZAMUDIO, P. 2014. Caracterización fisicoquímica, reológica y funcional de harina de avena (Avena sativa L. cv bachiniva) cultivada en la región de Cuauhtémoc, Chihuahua. Tecnociencias. 8(3):152-162.

14. GARCÍA, A.; PÉREZ, E.; DÁVILA, R. 2012. Características físicas, químicas y funcionales de las harinas obtenidas por secano del ñame, ocumo y mapuey. Agronomía Tropical. 62(1-4):051-068.

15. GRANADOS, C.; GUZMAN, L.; ACEVEDO, D.; DÍAZ, M.; HERRERA, A. 2014. Propiedades funcionales del almidón de sagu (Maranta arundinacea). Biotecnología Sector Agropecuario Y Agroindustrial. 12(2):90-96.

16. GUO, J.; LIU, L.; LIAN, X.; LI, L.; WU, H. 2014. The properties of different cultivars of Jinhai sweet potato starches in China. Internal J. Biological Macromolecules. 67:1-6. https://doi.org/10.1016/j.ijbiomac.2014.03.002

17. HERNÁNDEZ, M.; MONTES, A.; RODRÍGUEZ, B.; HERNÁNDEZ, L.; MEDINA, C.; RÍOS, D.; RODRÍGUEZ, E.; DIAZ, C. 2016. Application of multidimensional scaling technique to differentiate sweet potato (Ipomoea batatas (L.) Lam) cultivars according to their chemical composition. J. Food Composition and Analysis. 46:43-49. https://doi. org/10.1016/j.jfca.2015.10.008

18. HENAO, S.; ARISTIZÁBAL, J. 2009. Influencia de la variedad de yuca y nivel de sustitución de harinas compuestas sobre el comportamiento reológico en panificación. Ingeniería e investigación. 29(1):39-46.

19. JULIANTI, E.; RUSMARILIN, H.; YUSRAINI, E. 2015. Functional and rheological properties of composite flour from sweet potato, maize, soybean and xanthan gum. J. Saudi Soc. Agricultural Sciences. 2(16):171-177. https:// doi.org/10.1016/j.jssas.2015.05.005

20. LEE, J.; CHO, A.; HONG, J.; PARK, D.; LIM, S. 2012. Physical properties of wheat flour composites dry-coated with microparticulated soybean hulls and rice flour and their use for low-fat doughnut preparation. J. Cereal Science. 56(3):636-643. https://doi.org/10.1016/j.jcs.2012.08.011

21. LI, Q.; LI, D.; WANG, L.; ÖZKAN, N.; MAO, Z. 2010. Dynamic Viscoelastic Properties of Sweet Potato Studied y Dynamic Mechanical Analyzer. Carbohydrate Polymers. 79(3):520525. https://doi.org/10.1016/j.carbpol.2009.08.035
22. LUCAS, J.; QUINTERO, V.; CÁRDENAS, C. 2013. Caracterización de harina y almidón obtenidos a partir de plátano guineo AAAea (Musa sapientum L.). Acta Agronómica. 62(2):83-96.

23. MISHRA, S.; RAI, T. 2006. Morphology and functional properties of corn, potato and tapioca starches. Food hydrocolloids. 20(5):557-566. https://doi.org/10.1016/ j.foodhyd.2005.01.001

24. NASRIN, T.; ANAL, A. 2014. Resistant starch III from culled banana and its functional properties in fish oil emulsion. Food Hydrocolloids. 35(1):403-409. https://doi. $\operatorname{org} / 10.1016 /$ j.foodhyd.2013.06.019

25. PACHECO, E.; TECHEIRA, N. 2008. Propiedades químicas y funcionales del almidón nativo y modificado de ñame (Dioscorea alata). Revista Interciencia. 34 (4):280-285.

26. PATERNINA, A.; FIGUEROA, J.; SALCEDO, J.; CERVERA, M. 2016. Propiedades de empastamiento en almidones nativos de yuca, ñame y batata. Agronomía Colombiana. 34(1Supl):S402-S404. https://doi.org/10.15446/agron. colomb.sup.2016n1.58196

27. PRAMODRAO, K.; RIAR, C. 2014. Comparative study of effect of modification with ionic gums and dry heating on the physicochemical characteristic of potato, sweet potato and taro starches. Food Hydrocolloids. 35:613-619. https:// doi.org/10.1016/j.foodhyd.2013.08.006

28. RIVAS, M. 2012. Almidón acetilado de plátano: caracterización fisicoquímica y molecular. Tesis Doctoral en Ciencia de Alimentos. Facultad de Química. Universidad Autónoma de Querétaro. Querétaro (México). p118. Disponible desde Internet en: http://ri.uaq.mx/handle/123456789/1754 (con acceso el 14/05/2018).

29. ROJAS, E.; HOYOS, J.; MOSQUERA, S. 2016. Producción de polihidroxialcanoatos (PHAS) a partir de Ralstonia eutropha en un medio con harina de yuca como fuente de carbono. Biotecnología en el Sector Agropecuario y Agroindustrial. 14(1):19-26. https://doi.org/10.18684/BSAA(14)19-26

30. SACÓN, E.; BERNAL, I.; DUEÑAS, A.; COBEÑA, G.; LÓPEZ, N. 2016. Reología de mezclas de harinas de camote y trigo para elaborar pan. Tecnología Química. 35(3):457-467.

31. SALCEDO, J.; HERNÁNDEZ, E.; CERVERA, M.; BUELVAS, J. 2016. Evaluación de ocho clones de batata (Ipomoea batatas L.) para determinar su uso agroindustrial. Proc. Internal Agroindustrial Engineering Conference. 1:9-24.

32. TAPIA, D.; SOBRAL, P.; MENEGALLI, F. 2010. Potential of Amaranthus cruentus BRS Alegria in the production of 
flour, starch and protein concentrate: chemical, thermal and rheological characterization. J. Science of Food and Agriculture. 90(7):1185-1193. https://doi.org/10.1002/ jsfa.3946

33. TECHEIRA, N.; SÍVOLI, L.; PERDOMO, B.; RAMÍREZ, A.; SOSA, F. 2014. Caracterización fisicoquímica, funcional y nutricional de harinas crudas obtenidas a partir de diferentes variedades de yuca (Manihot esculenta Crantz), batata (Ipomoea batatas Lam) y ñame (Dioscorea alata), cultivadas en Venezuela. Interciencia. 39(3):191-197.

34. TECSON, E. 2007. Development of functional foods in the Philippines. Food Science and Technology Research. 13(3):179-186. https://doi.org/10.3136/fstr.13.179
35. VARGAS, P.; HERNÁNDEZ, D. 2012. Harinas y almidones de yuca, ñame, camote y ñampí: propiedades funcionales y posibles aplicaciones en la industria alimentaria. Tecnología en Marcha. 26(1):37-45.

36. YAACOB, B.; AMIN, M.; HASHIM, K.; BAKAR, B. 2011. Optimization of reaction conditions for carboxymethylated sago starch. Iranian Polymer J. 20(3):195-204

37. ZHOU, W.; YANG, J.; HONG, Y.; LIU, G.; ZHENG, J.; GU, Z; ZHANG, P. 2014. Impact of amylose content on starch physicochemical properties in transgenic sweet potato. Carbohydrate polymers. 122:417-427. https://doi. org/10.1016/j.carbpol.2014.11.003 